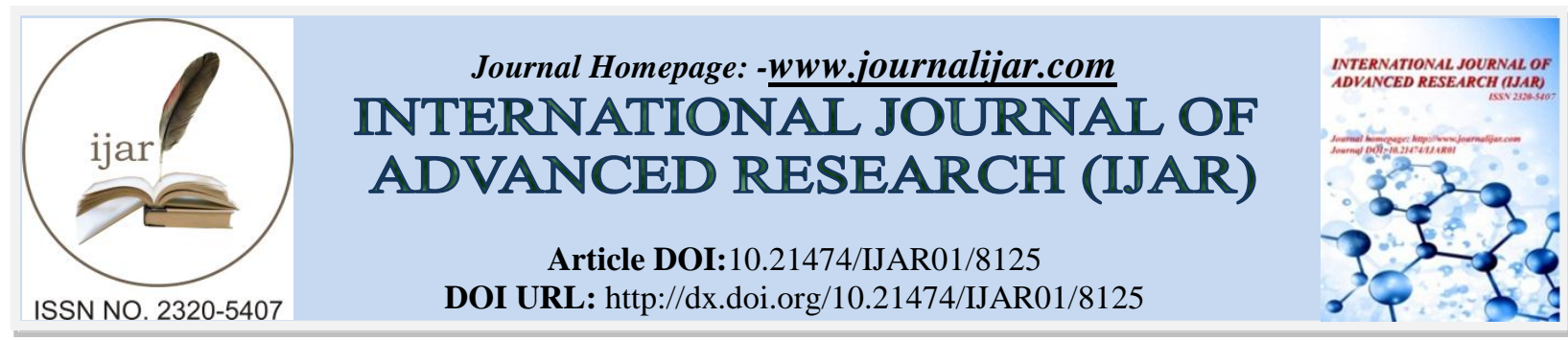

RESEARCH ARTICLE

\title{
GYNECOMASTIE UNILATERALE PERSISTANTE CHEZ LE PETIT ENFANT : A PROPOS D'UN CAS PERSISTENT UNILATERAL GYNECOMASTIA IN A SMALL CHILD: ONE CASE REPORT.
}

\author{
Abdelkoddous bhihi ${ }^{1}$, Moulay driss elamrani ${ }^{1}$, yassine benchamkha ${ }^{1}$, Aicha bourrahouat ${ }^{2}$, Ibtissam Khattou $^{2}$, \\ and Imane Ait $\mathbf{S a b}^{2}$. \\ 1. service de chirurgie plastique, réparatrice et esthétique.CHU Mohammed VI Marrakech. \\ 2. Service de pédiatrie B. CHU Mohammed VI Marrakech.
}

\section{Manuscript Info}

\section{Manuscript History}

Received: 01 October 2018

Final Accepted: 03 November 2018

Published: December 2018

Keywords:-

Breast, gynecomastia, child,

subcutaneous mastectomy.

\begin{abstract}
La gynécomastie est une hypertrophie mammaire chez l'homme, unilatérale ou bilatérale. Elle est secondaire à un déséquilibre hormonal entre androgènes et œstrogènes, au profit de ces derniers. Le plus souvent idiopathique et péripubertaire, elle justifie cependant un bilan étiologique complet, particulièrement pour écarter toute endocrinopathie ou étiologie maligne, avant d'envisager un éventuel traitement chirurgical en l'absence de réponse au traitement médical ou de régression spontanée. Dans cet article les auteurs rapportent un cas de gynécomastie persistante chez un petit enfant de 2 ans et demi suivi en consultation pédiatrique et au service de chirurgie plastique au CHU Mohamed VI Marrakech (Maroc).

Abstract : Gynecomastia is an enlarged breast in men, unilateral or bilateral caused by an imbalance of the hormones estrogen and testosterone. Most often idiopathic and peripubertal, it nevertheless justifies a complete etiological assessment to rule out endocrinopathy or a malignant etiology, before considering surgical treatment in the absence of response to medical treatment or spontaneous regression. In this article the authors report a case of persistent gynecomastia in a small child of two years and a half followed in pediatric consultation and the plastic surgery department at the University Hospital Mohamed VI Marrakech (Morocco).
\end{abstract}

Copy Right, IJAR, 2018,. All rights reserved.

\section{Introduction:-}

La gynécomastie unilatérale chez l'enfant est une affection rare avec quelques cas retrouvés dans la littérature. Elle nécessite un bilan étiologique détaillé avant d'évoquer son caractère idiopathique. Les auteurs rapportent un cas de gynécomastie unilatérale droite persistante chez un petit enfant de 2 ans et demi suivi en consultation pédiatrique et au service de chirurgie plastique au CHU Mohamed VI Marrakech (Maroc). Le diagnostic d'une gynécomastie idiopathique a été retenu devant un bilan étiologique qui s'est révélé normal. Devant l'absence d'amélioration spontanée de la masse, une chirurgie a été réalisée avec un bon résultat esthétique.

Corresponding Author:-Abdelkoddous bhihi.

Address:- service de chirurgie plastique, réparatrice et esthétique.CHU Mohammed VI Marrakech. 


\section{Observations:-}

Enfant de 02ans et demi, de sexe masculin, sans antécédents pathologiques particuliers et pas de cas similaires dans la famille, qui présente depuis un an une hypertrophie mammaire unilatérale droite, augmentant progressivement de volume sans autres signes associés notamment pas de mastodynie ni d'écoulement mammaire. L'examen clinique trouve une gynécomastie droite stade S2 sans écoulement ni altération du tissu cutané en regard (figure1) et les organes génitaux externes sont d'aspect normal. Le caryotype s'est avéré sans anomalie. Les explorations endocriniennes (TSH, testostérone, LH, FSH, Prolactine, œstradiol, HCG, alpha-foeto-proteine, fonction rénale, fonction hépatique) étaient normales. L'échographie mammaire a objectivé une hyperplasie droite unilatérale, d'aspect ovalaire, rétro-aréolaire, centrée sur le mamelon, mesurant $31 \times 11 \mathrm{~mm}$, en faveur d'une gynécomastie. Devant l'absence d'une étiologie déterminée et l'absence d'une régression spontanée de la masse, une exérèse chirurgicale a été réalisée. La technique chirurgicale a consisté en une mastectomie sous cutanée par voie hémiaréolaire inférieure, tout en respectant une épaisseur glandulaire en regard de la plaque aréolo-mammelonaire assurant sa vascularisation (figure 2). Une contention a été maintenue pendant 04 semaines. L'étude anatomopathologique était en faveur d'une gynécomastie sans signes histologiques de malignité.
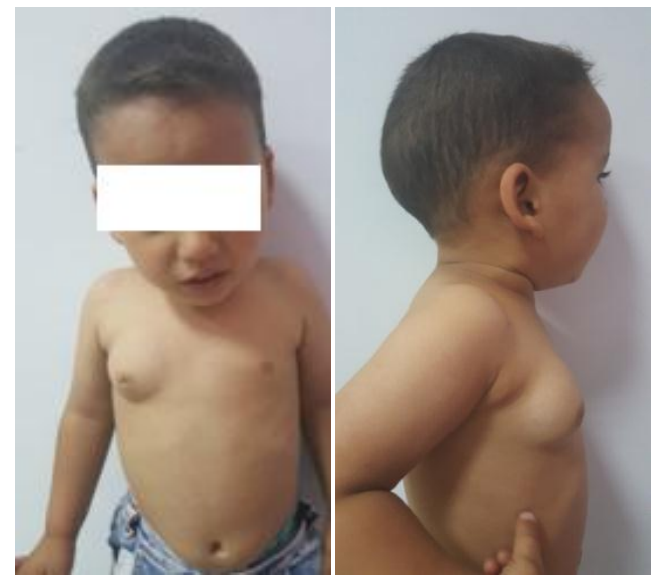

Figure 1:-Hypertrophie du sein droit stade S2 sans écoulement ni altération du tissu cutané en regard (a) vue de face (b) vue de profil.

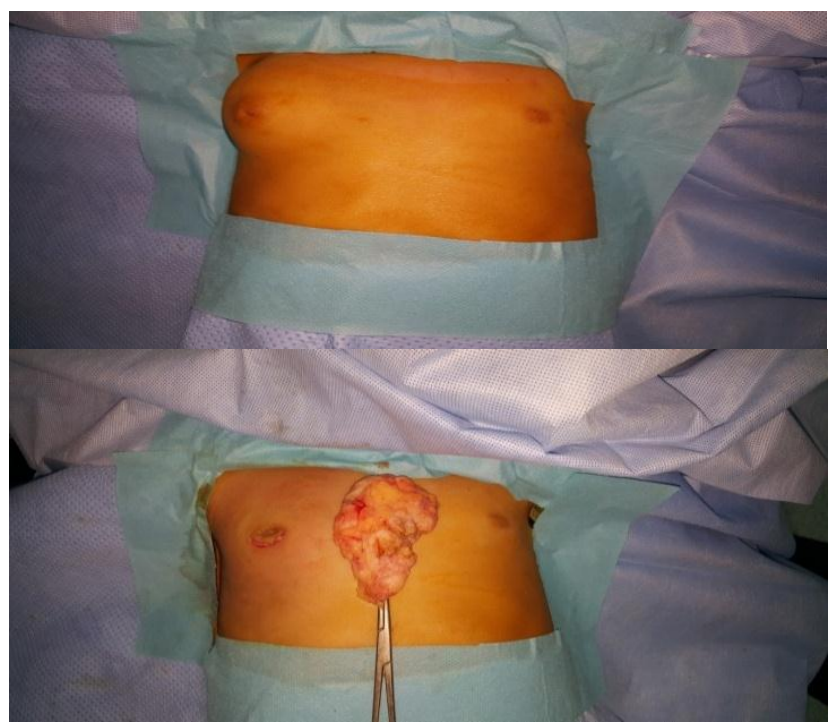

Figure 2:-Vue per opératoire 


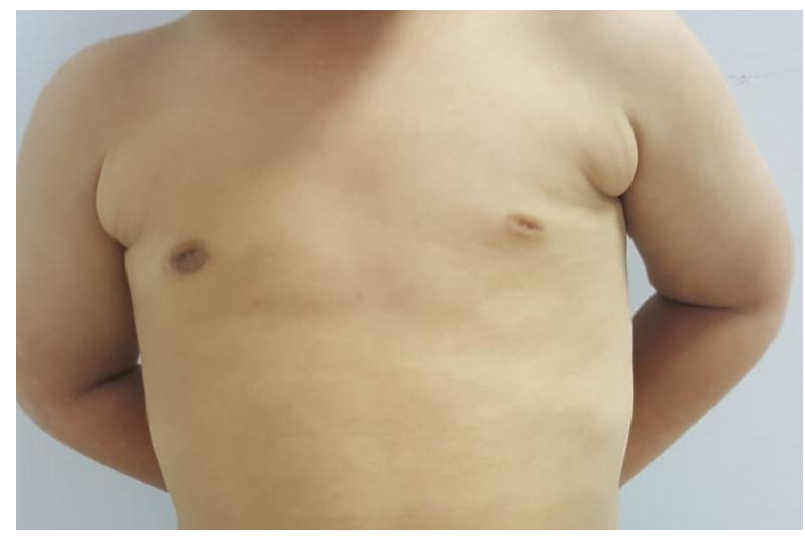

Figure 3:-résultat 5 mois après l'acte chirurgical.

\section{Discussion:-}

La gynécomastie se définit par une hyperplasie uni- ou bilatérale, non tumorale de la glande mammaire chez l'homme. La gynécomastie chez les garçons durant les premiers mois de vie est fréquente, elle est due à la persistance d'un taux d'œstradiol circulant d'origine maternelle. La taille des seins est corrélée à ce taux d'œstradiol qui est élevé surtout durant les 03 premiers mois [1]. A la puberté, la gynécomastie est fréquente et se présente sous forme d'une masse sous aréolaire ou une augmentation diffuse du volume de la glande mammaire. Elles sont majoritairement idiopathiques sans cause évidente et elles ont tendance à une régression spontanée [2]. Avant la puberté, la gynécomastie est rare et le diagnostic différentiel se pose avec des tumeurs sécrétant HCG et œstrogène, le syndrome des hyperaomatases, l'hyperthyroidie, l'usage de drogues [3].

La gynécomastie unilatérale chez l'enfant reste une pathologie rare imposant un bilan spécialisé à la recherche d'une étiologie avant d'envisager un traitement chirurgical malgré que plus de 50\% des cas restent sans cause évidente [4]. Dans le cas de notre patient, l'avis médical a été demandé devant l'absence d'une régression spontanée et l'inquiétude parentale du retentissement négatif de cette pathologie sur le développement psychologique de leur enfant. La relation entre gynécomastie, un mauvais état mental et une faible estime de soi a été démontrée [5].

Chez notre patient il n'y a pas de notion de prise médicamenteuse ni autres causes iatrogènes et le bilan biologique réalisé était normal ce qui nous a permis d'écarter toute endocrinopathie ou étiologie maligne avant d'envisager une exérèse chirurgicale. Le traitement chirurgical de gynécomastie peut être réalisé en utilisant plusieurs techniques [6,7]. Dans le cas de notre patient, on a opté pour une mastectomie sous cutanée tout en gardant une épaisseur glandulaire en regard de la plaque aréolo-mammelonnaire assurant sa vascularisation. Cette plaque a tendance à se rétracter avec le temps pour rejoindre en taille le coté controlatéral normal.

En post-opératoire, les complications immédiates sont dominées par les hémorragies et les hématomes d'où l'intérêt d'une contention qui doit être maintenue pendant 04 à 08 semaines. Les complications secondaires sont surtout d'ordre esthétique. Chez notre patient le résultat esthétique a été jugé très satisfaisant par l'équipe soignante ainsi que les parents (figure 3). Les enfants porteurs d'une gynécomastie présentent une mauvaise qualité de vie en comparaison avec des sujets normaux de leur âge [8,9], l'apport de la chirurgie dans l'amélioration de la qualité de vie a été prouvé permettant aux patients de retrouver un état psychosocial normal [10]. Dans notre cas, la prise en charge chirurgicale précoce avant la scolarisation assurera à notre patient une vie sociale normale en lui permettant de retrouver une silhouette masculine sans ambiguité.

\section{Conclusion:-}

Nous rapportons ce cas pour souligner l'importance d'une enquête étiologique détaillée avant de conclure à une gynécomastie idiopathique et l'intérêt de traitement chirurgical dans la prévention de l'altération de l'acquisition du schéma corporelle chez l'enfant.

\section{Déclaration d'intérêts:-}

Les auteurs déclarent ne pas avoir de conflits d'intérêts en relation avec cet article 


\section{Références:-}

1. Arca MJ, Caniano DA. Breast disorders in the adolescent patient. Adolesc Med Clin 2004;15:473-85.

2. Durkin ET, Warner TF, Nichol PF. Enlarging unilateral breast mass in an adolescent male: an unusual presentation of intraductal papilloma.J Pediatr Surg 2011;46:33-5.

3. Henley DV, Lipson N, Korach KS, et al. Prepubertal gynecomastia linked to lavender and tea tree oils. N Engl J Med 2007;356:479-85.

4. Derkacz M, Chmiel-Perzynska I, Nowakowski A. Gynecomastia - a difficult diag-nostic problem. Endokrynol Pol 2011;62:190-202.

5. Ordaz DL, Thompson JK. Gynecomastia and psychological functioning: A review of the literature. Body Image 2015;15:141-8.

6. Nordt CA, DiVasta AD. Gynecomastia in adolescents. Curr Opin Pediatr 2008;20:375-82.

7. Wong KY, Malata CM. Conventional versus ultrasound-assisted liposuctionin gynecomastia surgery: a 13-year review. J Plast Reconstr Aesthet Surg2014;67:921-6.

8. Kinsella Jr. C, Landfair A, Rottgers SA, et al. The psychological burden of idio-pathic adolescent gynecomastia. Plast Reconstr Surg 2012;129:1-7.

9. Ordaz DL, Thompson JK. Gynecomastia and psychological functioning: A review of the literature. Body Image 2015;15:141-8.

10. L.C. Nuzzi et al. TheEffect of Surgical Treatment for Gynecomastia on Quality of Life in Adolescents. Journal of Adolescent Health 00 (2018). 